\title{
Genomic profile of ovarian carcinomas
}

\author{
Francesca Micci ${ }^{1,2^{*}}$, Lisbeth Haugom,2, Vera M Abeler ${ }^{3}$, Ben Davidson ${ }^{3,4}$, Claes G Tropé ${ }^{5}$ and Sverre Heim ${ }^{1,2,4}$
}

\begin{abstract}
Background: It is known that all tumors studied in sufficient number to draw conclusions show characteristic/ specific chromosomal rearrangements, and the identification of these chromosomes and the genes rearranged behind the aberrations may ultimately lead to a tailor-made therapy for each cancer patient. Knowledge about the acquired genomic aberrations of ovarian carcinomas is still unsatisfactory.

Methods: We cytogenetically analyzed 110 new cases of ovarian carcinoma of different histological subtypes using karyotyping of G-banded chromosomes and high-resolution comparative genomic hybridization. We first compared the aberration patterns identified by the two genomic screening techniques using the so-called "classical" pathological classification in which the carcinomas are grouped as tumors of types I and II. We also broke down our findings according to the more "modern" classification which groups the carcinomas in five different categories.

Results: The chromosomal breakpoints identified by karyotyping tended to cluster to $19 \mathrm{p} / \mathrm{q}$ and to $11 \mathrm{q}$, but no unquestionably recurrent rearrangement could be seen. Common imbalances were scored as gains from 1q, 3q, $7 q$, and $8 q$ and losses from 17p, 19q, and 22q. Gain of material from 8q23 and losses from 19q and 22q have previously been described at high frequencies in bilateral and borderline ovarian carcinomas. The fact that they were present both in "precursor" lesions, i.e., borderline tumors, as well as in tumors of more advanced stages, i.e., carcinomas, highlights the possibility of an adenoma-carcinoma sequence in ovarian carcinogenesis.

Conclusion: Based on the relatively simple genomic changes we identified in the low-grade serous carcinomas examined $(n=7)$ and which largely corresponded to the aberration pattern formerly identified in borderline tumors, one can interpret the cytogenetic data as supporting the view that the low-grade carcinomas represent a phenotypically more advanced stage of borderline tumors. Whether transition from low-grade to high-grade carcinoma also occurs, is a question about which the genomic data is still inconclusive.
\end{abstract}

Keywords: Ovarian carcinoma, Karyotyping, $\mathrm{HR}-\mathrm{CGH}$

\section{Background}

Cancer of the ovary represents $30 \%$ of all cancer of the female genital organs [1]. Adenocarcinomas make up $75 \%$ of all ovarian tumors and $95 \%$ of ovarian malignancies [1]. At the same time, surface epithelial tumors of the ovary display a wide variety of histologic types which may reflect pathogenetic variability and/or differences among the cell(s) of origin. One potentially useful way of classifying these neoplasms is to divide them into those that appear to develop from benign and/or atypical proliferative precursor lesions (mucinous, endometrioid,

\footnotetext{
* Correspondence: francesca.micci@labmed.uio.no

'Section for Cancer Cytogenetics, Institute for Cancer Genetics and Informatics, The Norwegian Radium Hospital, Oslo University Hospital, 0310 Oslo, Norway

${ }^{2}$ Centre for Cancer Biomedicine, University of Oslo, Oslo, Norway

Full list of author information is available at the end of the article
}

clear cell, and low-grade serous carcinomas, also referred to as type I tumors) and those that appear to develop de novo (high-grade serous carcinomas or type II tumors) $[2,3]$. However, the apparent difference between serous and nonserous tumors in this regard may reflect the rapidity of the adenoma-carcinoma transition rather than any qualitative pathogenetic difference. Specifically, it can be argued that the adenoma-carcinoma sequence for serous tumors is variable but, on average, shorter than that for mucinous, endometrioid, and clear-cell tumors; this would explain the relative uniformity of serous carcinomas and the infrequent presence in them of neoplastic precursor lesions [2]. It is obvious that use of the term "de novo" in this context reflects our ignorance about the early events of ovarian carcinogenesis rather than real insight into the tumor origin.

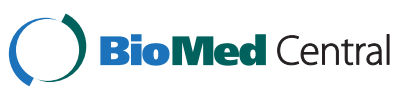

(c) 2014 Micci et al.; licensee BioMed Central Ltd. This is an Open Access article distributed under the terms of the Creative Commons Attribution License (http://creativecommons.org/licenses/by/2.0), which permits unrestricted use, distribution, and reproduction in any medium, provided the original work is properly credited. The Creative Commons Public Domain Dedication waiver (http://creativecommons.org/publicdomain/zero/1.0/) applies to the data made available in this article, unless otherwise stated. 
At the same time, pathologists also classify ovarian carcinomas into five main types based on histopathological, immunohistochemical, and molecular genetic findings: high-grade serous carcinomas, endometrioid carcinomas, clear-cell carcinomas, mucinous carcinomas, and low-grade serous carcinomas [4]. The tumors belonging to these categories account for $98 \%$ of ovarian carcinomas. They differ group-wise from one another also with regard to epidemiological and genetic risk factors, precursor lesions, patterns of spreading, molecular events during oncogenesis, response to chemotherapy, and prognosis [4].

Many of the more than 400 (at the moment 438) scientifically reported ovarian carcinomas with karyotypically characterized chromosomal aberrations [5] were examined as abdominal effusions, that is at a very late stage in tumor progression, and many were incompletely karyotyped. Knowledge about the chromosomal characteristics of this type of cancer is therefore far from complete.

We here present 110 new cases of ovarian carcinoma of different histologic subtypes (73 high-grade serous carcinomas, seven low-grade serous, 15 endometrioid, eight clear-cell, three mucinous, three mixed endometrioid and mucinous, and one undifferentiated carcinoma) whose genome was characterized by G-banding followed by karyotyping and high-resolution comparative genomic hybridization (HR-CGH). We specifically compared the acquired genomic aberration pattern of the high-grade serous carcinomas with that of the other types in order to address the question if they share common genomic abnormalities and therefore have a common/similar pathogenesis or, alternatively, if the high-grade serous tumors warrant being considered as a wholly distinct entity. We also scrutinized the aberration patterns of the five different subgroups to identify whatever genomic aberrations might distinguish each group or if any are common to two or more ovarian carcinoma types.

\section{Methods}

\section{Tumors}

The material consisted of fresh samples from 110 primary ovarian carcinomas surgically removed at The Norwegian Radium Hospital from 1999 to 2004 (Additional file 1: Table S1). Neoadjuvant therapy had been given in 25 of the cases before surgery (Additional file 1: Table S1). The tumors were classified as high-grade serous adenocarcinoma (73 samples), low-grade serous adenocarcinoma (seven samples), endometrioid carcinoma (15 samples), clear-cell carcinoma (eight samples), mucinous carcinoma (three samples), undifferentiated carcinoma (one sample), and mixed histology between endometroid and mucinous carcinoma (three samples and cases).
The project has been approved by the Regional Ethics Committee for Medical and Health Research Ethics SouthEast in the year 2007 (project number 2.2007.425). Written informed consent was obtained from the patients. Frozen tissue was retrieved from The Radium Hospital biobank (nr 1971, S-07194a approved by the Regional Ethics Committee and The Directory of Health (Helsedirektorat) in 2007). The entire study was also approved by the institutional review board at the Norwegian Radium Hospital.

\section{Cell culturing and karyotyping}

The tumor samples were manually minced and disaggregated with Collagenase II (Worthington, Freehold, NJ, USA) until a suitable suspension of cells and cell clumps was obtained. After 6-7 days of culturing in a selective medium [6], colchicine was added and the cultures harvested according to Mandahl [7]. The chromosomes of the dividing cells were then G-banded and a karyotype established according to the recommendations of the ISCN [8].

\section{High-resolution comparative genomic hybridization (HR-CGH)}

DNA was isolated by the phenol-chloroform method as previously described [9]. CGH was performed according to our modifications of standard procedures [10,11]. Chromosomes were karyotyped based on their inverted DAPI appearance and the relative hybridization signal intensity was determined along each chromosome. On average, 10-15 metaphases were analyzed. The HR-CGH analysis was performed as previously described [12]. The description of the CGH copy number changes was based on the recommendation of the ISCN [8].

\section{Results}

Karyotypic information was obtained on 86 of the original 110 samples (Additional file 1: Table S1). The 24 cultures that failed showed either no cell attachment after several days in vitro or no metaphases. A normal karyotype was found in 22 tumors. The remaining 64 tumors (Additional file 1: Table S1) mostly showed complex karyotypes with several structural and numerical aberrations. Simple karyotypes, by which we mean karyotypes showing less than four aberrations, were seen only in one high-grade serous carcinoma (case 35), two low-grade serous carcinomas (cases 77 and 78), one clear-cell carcinoma (case 102), and one mixed endometrioid and mucinous carcinoma (case 107). Case 35 (a high-grade serous tumor) showed monosomies of chromosomes $\mathrm{X}, 4$, and 10. Case 77 (a low-grade serous tumor) showed two unrelated clones with monosomy 11 and monosomy of the $\mathrm{X}$ chromosome, respectively. Case 78 (a low-grade serous tumor) showed trisomy 7 as the sole abnormality in all cells analyzed. Case 102 (a clear-cell tumor) had 
$\mathbf{A}_{100 \%}$

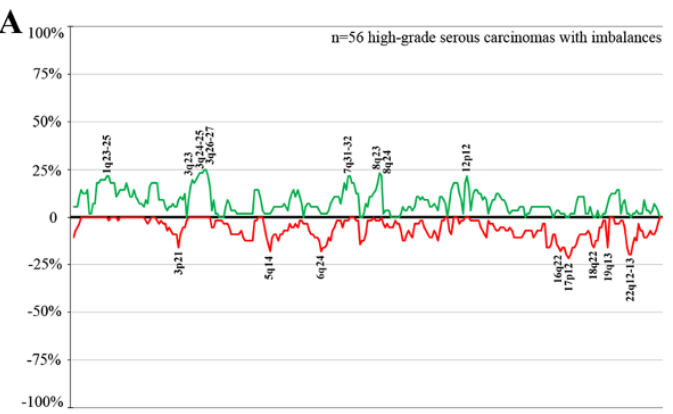

B

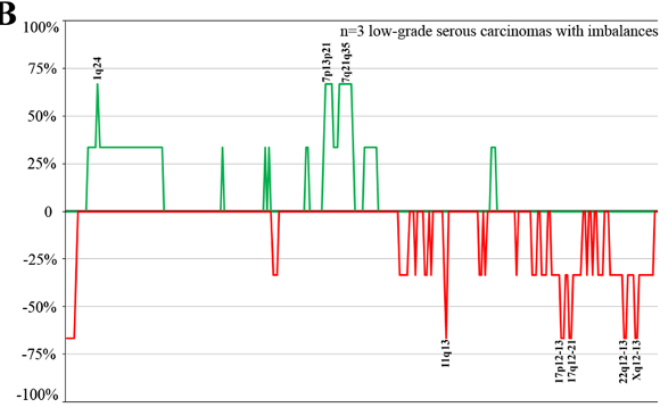

$\mathbf{C}_{100}$
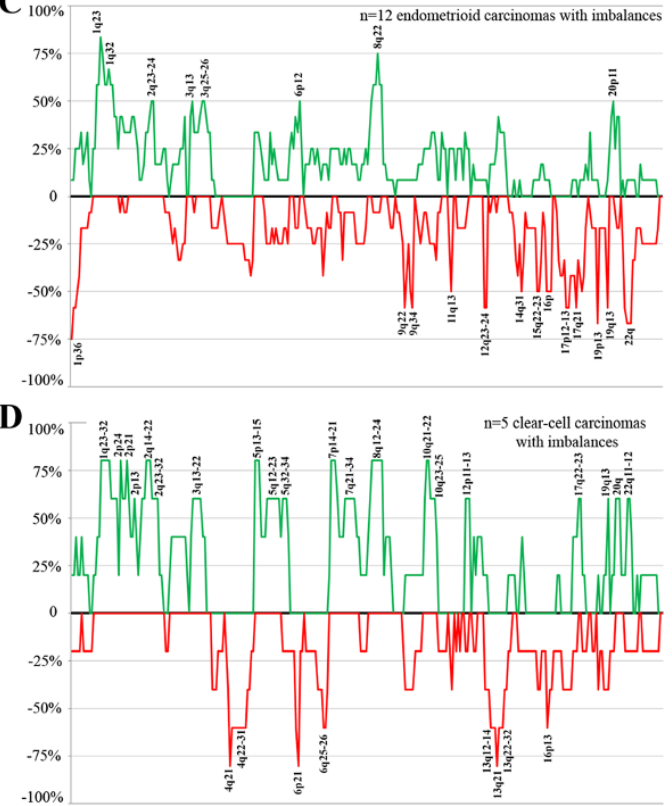

$\mathbf{E}_{100}$

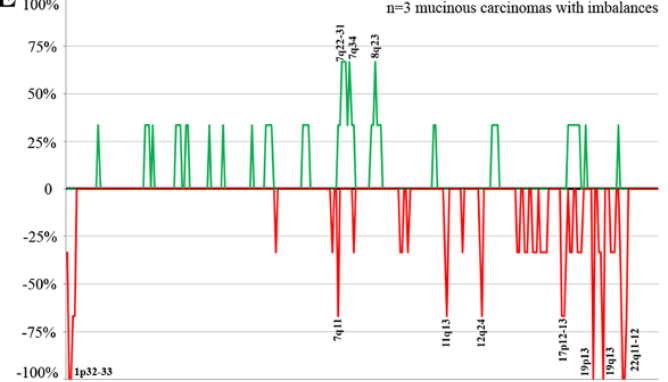

Figure 1 Profiles of the imbalances detected by HR-CGH in the different subtypes of ovarian carcinomas. The green color highlights gains, while the red color indicates losses. A) high-grade serous carcinomas; B) low-grade serous carcinomas; C) endometrioid carcinomas; D) clear-cell carcinomas; and E) mucinous carcinomas.

two supernumerary markers of unknown origin in three metaphases of the 45 cells analyzed. Case 107 (also a mixed endometrioid and mucinous carcinoma) showed additional material of unknown origin on $8 \mathrm{q} 24$ as the sole chromosomal abnormality. The only tumor showing obvious clonal evolution (i.e., beyond having several aberrations) was case 38 (a high-grade serous carcinoma) which had three related clones with only numerical aberrations corresponding to the karyotype $47 \sim 49, \mathrm{XX},+8,+9[2] / 49$, idem, $+5,-6,+7[4] / 54$, idem $,+3,+5,+6,+7,+14,+17,+19[5]$. Breakpoint clusters were seen at chromosome arms 11q (33 out of altogether 431 breaks, 7.65\%), 19p (27 breaks; $6.26 \%$ ), and 19q (22 breaks; 5.1\%). The aberrations involving these chromosomes were of different types, e.g., deletions, translocations, and additions of unknown material. Although simple rearrangements between two chromosomes could be described in some tumors, in the majority of cases it was not possible to identify the partner chromosome in the rearrangement which led us to resort to descriptions such as $\operatorname{add}(11)(\mathrm{p} / \mathrm{q})$ and/or $\operatorname{add}(19)(\mathrm{p} / \mathrm{q})$.

The HR-CGH analysis of genomic imbalances showed gains and/or losses in 81 tumors (Additional file 1: Table S1), whereas no imbalances were scored in seven samples. No informative results were obtained in another seven tumors due to poor quality of hybridization signals. In the remaining 15 cases, no DNA was available to perform the analysis. Gains were more frequent than losses and amplifications, i.e., more than four-fold gains, were scored in 30 tumors (21 high-grade serous carcinomas, four endometrioid, two clear-cell, one low-grade serous, one mucinous, and one undifferentiated carcinoma). The high-grade serous carcinomas ( $\mathrm{n}=56$ with imbalances) mostly showed gains of 3q26-27 (detected in $25 \%$ of the tumors with imbalances), followed by gains of 3q24-25 and $8 \mathrm{q} 23$ (in 23\%), and 1q31-32, 3q23, 3q28, 7q31-32, $8 \mathrm{q} 24$, and $12 \mathrm{p} 12$ (all detected in $21 \%$ of the tumors; Figure 1). The most frequent losses were scored at $17 \mathrm{p} 12$ (21\%), 17p11, 17p13, and 22q12-13 (20\%), and 5q14, 6q24, and 16q22 (18\%). The endometrioid carcinomas $(\mathrm{n}=12$ with imbalances) showed gains of 1q23 (83\% of the endometrioid tumors with imbalances), followed by $1 \mathrm{q} 24$ and $8 \mathrm{q} 22$ (75\%), 1q32 (67\%), 1q21-22, 1q25-31, 1q41-42, $8 \mathrm{q} 13-21$, and $8 \mathrm{q} 23 \mathrm{q} 24$ (58\%), and 2q23-24, 3q13, 3q25-26, $6 \mathrm{p} 12,8 \mathrm{q} 12$, and 20p11 (50\%; Figure 1). Losses were scored at $1 \mathrm{p} 36(75 \%)$ followed by $19 \mathrm{p} 13$ and $22 \mathrm{q}(67 \%)$, and $9 \mathrm{q} 22$, 9q34, 12q23-24, 17p12-13, 17q21, 19q13, and 21q22 
(58\%). The clear-cell carcinomas ( $\mathrm{n}=5$ tumors with imbalances) showed frequent gains from 1q23-32, 2p24, 2p21, 2q14-22, 5p13-15, 7p14-21, 8q12-24, and 10q21-22 (80\%) followed by 1q41-44, 2p22-23, 2p13, 2q12-13, 2q2332, 3q13-24, 5q12-23, 5q32-34, 7p13, 7q21-34, 8q11, 10q11, 10q23-25, 12p11-13, 17q22-23, 19q13, 20q, and 22q11-12 (60\%). Losses were most often scored at 4q21, $6 \mathrm{p} 21$, and 13q21 (80\%) followed by 4q22-31, 6p22, 6q2526, 13q12-14, 13q22-32, and 16p13 (60\%; Figure 1). The mucinous carcinomas ( $\mathrm{n}=3$ with imbalances) showed frequent gains of $7 q 22-31,7 q 34$, and $8 q 23$ (67\%). Losses were scored at $19 \mathrm{p} 13,19 \mathrm{q} 13$, and $22 \mathrm{q} 11-12$ in all three tumors with imbalances (100\%) followed by losses of 7q11, 11q13, 12q24, 17p12-13, and 22q13 (67\%; Figure 1). The low-grade serous carcinomas $(n=3$ with imbalances) had gains mostly of 1q24, 7p13-21, and 7q21-35 (67\%), while losses were commonly detected at 11q13,17p12-13,17q12-21, 22q12-13, and Xq12-13 (67\%; Figure 1). Amplifications most frequently involved chromosomal bands 3q26 (12 tumors) followed by 8q23 (11 tumors), 8q22 (ten tumors), 8q24 (eight tumors), and 3q25 and 8q21 (seven tumors). The high-grade serous carcinomas showed amplifications in 3q26 in ten samples followed by $8 \mathrm{q} 23(\mathrm{n}=9), 8 \mathrm{q} 22$ $(\mathrm{n}=8), 8 \mathrm{q} 24(\mathrm{n}=7)$, and $3 \mathrm{q} 25$ and $8 \mathrm{q} 21$ in six samples each. Four endometrioid carcinomas showed amplification, case 84. (Additional file 1: Table S1) in 3q26 and 12 , case 85 in 1q42-44, case 90 in 5p14, and case 93 in 3q25-29 and 6p21-22. Two clear-cell carcinomas showed amplifications: case 97 in $8 \mathrm{q}$ and case 100 in 8q13-21 and 8q22-23. The only low-grade serous carcinoma showing amplification (case 75) had it in 1q31, the undifferentiated carcinoma (case 110) showed amplification in 12q13, and a mucinous carcinoma (case 104) had 7q31 amplified.

Both tumors that had received $(n=15)$ and those that had not received $(n=41)$ neo-adjuvant therapy prior to the operation showed frequent gains from $3 q$ and $7 q$; however, the neo-adjuvant group also showed gain of 11 14-22 in $27 \%$ of the abnormal cases, whereas the group that did not receive such treatment showed common additional gains from 1q, 2q, 8q, and 12p. Losses were often seen at $8 \mathrm{p}, 9 \mathrm{q}$, and $10 \mathrm{q}$ in the neo-adjuvant subgroup but more commonly at $3 \mathrm{p}, 5 \mathrm{q}, 6 \mathrm{q}, 17 \mathrm{p}, 17 \mathrm{q}$, and $22 \mathrm{q}$ in the normal group.

The average number of copy aberrations (ANCA) index was 39.7 for the high-grade serous carcinomas, 14.7 for the low-grade serous carcinomas, 33.7 for the endometrioid carcinomas, 35.2 for the clear-cell carcinomas, and 20.3 for the mucinous carcinomas. The ANCA index for the tumors (all subtypes taken together) that had received neoadjuvant treatment was 30.85 whereas the value for the group of tumors without neoadjuvant treatment was 39.01 .

\section{Discussion}

We report the analysis of 110 ovarian carcinomas of different histological types using G-banding and HR-CGH. We first compared the aberration patterns identified by the two genomic screening techniques using the so-called "classical" pathological classification of ovarian carcinomas advocated by Blaustein [2] where the carcinomas are divided into tumors of types I and II. We also broke down our findings according to the more "modern" classification proposed by Prat [4], which classifies the carcinomas into five different categories.

The overall genomic complexity of the tumors could be assessed both by karyotyping and CGH. The acquired aberrations detected by G-banding resulted in complex karyotypes in the great majority of both type I and type II ovarian carcinomas. Breakpoint clusters were seen first and foremost at 11q, 19p, and 19q. A total of five rearrangements between chromosomes 11 and 19 (in altogether three tumors) were detected in this series; these have been examined in detail previously and reported by us [6]. In our experience with ovarian cancer karyotyping, this is the most common exchange of material between two chromosomes detectable with this technique. Lately, Onkes et al. [13] described a fusion gene corresponding to a $\operatorname{der}(19) \mathrm{t}(11 ; 19)(\mathrm{q} 13.2 ; \mathrm{p} 13.2)$ in the ovarian cell line SKOV3. The translocation led to a fusion between the Homo sapiens hook homolog 2 (HOOK2), mapping on $19 \mathrm{p}$, and the actinin alpha 3 (ACTN3), mapping on 11q. We have tested, using PCRbased methods, all 110 samples of the present series for this fusion whose chromosome-level rearrangement could possibly be hidden in the complex and only partially described karyotypes. However, we found no expression of this fusion gene in any of the tumors (data not shown). Possibly other genes mapping to $11 \mathrm{q}$ and $19 \mathrm{p}$ are involved in fusions; this needs to be further investigated.

With or without recombination with 11q, alterations of chromosome bands $19 \mathrm{p} 13$ and $19 \mathrm{q} 13$ are among the most frequent cytogenetic changes observed in ovarian carcinomas as first pointed out by Pejovic et al. [14], Jenkins et al. [15], and Thompson et al. [16]. Much later, we subjected a group of ovarian carcinomas showing additional material of unknown origin sitting on $19 \mathrm{p} / \mathrm{q}$ to examination by microdissection followed by reverse in situ hybridization and were able to identify the nature of the rearrangements in more detail [6]. We also performed arrayCGH to identify the genomic regions that were gained or amplified on the structurally rearranged $19 p / 19 q$ [17]. However, much more remains to be done to understand how the nonrandom $19 \mathrm{p} / \mathrm{q}$-changes contribute to ovarian carcinogenesis.

There was no difference in breakpoint clusters among the various histologic subtypes, i.e., they all had tumors 
with breakpoints clustering to $11 \mathrm{q}, 19 \mathrm{p}$, and $19 \mathrm{q}$. One should be cognizant of the caveat, however, that the great complexity of the rearrangements, as illustrated by the presence of many markers in most karyotypes, may have hidden rearrangements that could possibly distinguish between the different tumor subtypes. When we looked specifically at the serous subtype, we saw that the low-grade tumors $(n=7)$ with informative karyotypes $(n=5)$ were either cytogenetically normal (cases 79 and 80) or had only simple karyotypic aberrations (less than four per karyotype in cases 76, 77, and 78). On the other hand, the high-grade serous carcinomas $(n=73)$ showed mostly complex and/or incomplete karyotypes if they were not cytogenetically normal, the only exception being case 35 which showed monosomies for chromosomes X, 4, and 10 in three cells. These results are in accordance with the findings in a previous study of ours [12] in which we analyzed borderline ovarian carcinomas of the serous and mucinous subtypes and found only simple karyotypic changes. Furthermore, the results support the suggestion of Prat [4] that low-grade serous carcinomas can be defined as uncommon ovarian carcinomas that show a noninvasive serous borderline component, and that they most likely reflect progression of serous borderline tumors beyond microinvasion.

The most reliable assessment of genomic complexity in terms of gains/losses of chromosomal material was obtained by HR-CGH. We compared tumor subsets according to their ANCA index which was 29.8 for type I and 39.7 for type II carcinomas. The Mann-Whitney U-test showed a significant difference between the two groups $(\mathrm{p}<0.05$; Table 1$)$. The same test was also performed to see if there were significant differences in genomic complexity among the histologic subtypes (Table 1). The only significant difference was found between high- and low-grade serous carcinomas, with the latter tumors on average showing fewer genomic imbalances (ANCA index 39.7 vs 14.7). Obviously, larger sets of samples for each specific subtype should be tested to confirm or refute this conclusion. The same non-parametric test was also performed to see if there was a difference in the number of acquired chromosomal aberrations between tumors that had received $(n=15)$ or had not received $(n=41)$ neo-adjuvant therapy prior to the operation. There was no statistical significance $(\mathrm{p}=0.29)$. However, the Mann-Witney U-test did show a significant difference $(p=0.04)$ between the number of copy number changes, i.e., the ANCA index, in the two groups.

The imbalance patterns detected by HR-CGH were also assessed group-wise with comparisons between type I and type II tumors as well as among all five histologic carcinoma subtypes. The high-grade serous carcinomas (type II) mostly showed gains from 1q, 3q, 7q, 8q, and 12 p but losses from 3p, 5q, 6q, 16q, 17p, 18q, 19q, and $22 q$, whereas the low-grade tumors (i.e., the remainder) showed gains from $1 \mathrm{q}, 2 \mathrm{q}, 5 \mathrm{q}$, and $8 \mathrm{q}$ whereas losses were seen primarily from $1 \mathrm{p}, 9 \mathrm{q}, 11 \mathrm{q}, 12 \mathrm{q}, 16 \mathrm{p}, 17 \mathrm{p}$, 19 p, $19 q$, and 22q. Common imbalances to both type I and type II were gains from 1q, 3q, 7q and $8 \mathrm{q}$ and losses from $17 \mathrm{p}, 19 \mathrm{q}$, and $22 \mathrm{q}$. These results are in agreement with the result of previous imbalance studies of ovarian carcinomas [18-24] as well as with the latest research work performed by the Cancer Genome Atlas Research Network [25] on more than 400 ovarian carcinomas. Furthermore, gain of material from 8q23 and losses from $19 \mathrm{q}$ and $22 \mathrm{q}$ have been found at high frequencies in bilateral ovarian carcinomas where it was suggested that they probably represent early genomic changes because they were present in tumors of both sides [18]. The very same imbalances were also identified in borderline ovarian carcinomas [12]. The fact that they were present both in "precursor" lesions, i.e., borderline tumors, as well as more advanced stages, i.e., carcinomas, highlights the possibility of an adenoma-carcinoma sequence in ovarian carcinogenesis. The relationship between borderline ovarian tumors and low-grade carcinomas has been investigated at the gene level by many groups [26-28] focusing on the mutually exclusive mutations detected in either the $B R A F$ or KRAS gene, and a relationship was found between the two groups of tumors. We did not perform any molecular study on our cases; however, our chromosomal results fit the interpretation that a common relationship may exist between the two groups of tumors at the level of both genes and chromosomes.

When looking separately at the imbalance patterns of different subtypes of low-grade tumors, we saw examples of gains and/or losses shared by two or more subtypes

Table 1 Overview of the $p$ values obteined by comparing the ANCA index of the different histological subtypes of ovarian carcinomas, using the Mann-Whitney U-test

\begin{tabular}{|c|c|c|c|c|c|}
\hline & High-grade serous & Low-grade serous & Endometrioid & Clear cell & Mucinous \\
\hline High-grade serous & - & $p=0.03372$ & $p=0.2772$ & $p=0.624$ & $p=0.0874$ \\
\hline Low-grade serous & $p=0.03372$ & - & $p=0.1486$ & $p=0.1771$ & $p=0.5066$ \\
\hline Endometrioid & $p=0.2772$ & $p=0.1486$ & - & $p=0.8329$ & $p=0.3648$ \\
\hline Clear cell & $p=0.624$ & $p=0.1771$ & $p=0.8329$ & - & $p=0.2938$ \\
\hline Mucinous & $p=0.0874$ & $p=0.5066$ & $p=0.3648$ & $p=0.2938$ & - \\
\hline
\end{tabular}


but also copy number changes that were only or predominantly present in one subgroup, namely -Xq mostly in low-grade serous carcinomas, $+6 \mathrm{p},+20 \mathrm{p}$, and $-21 \mathrm{q}$ mostly in endometrioid carcinomas, and $+2 \mathrm{p},+5 \mathrm{p},+5 \mathrm{q},+10 \mathrm{q}$, $+12 \mathrm{p},+17 \mathrm{q},+19 \mathrm{q},+20 \mathrm{q},-4 \mathrm{q},-6 \mathrm{p}$, and $-13 \mathrm{q}$ mostly in clear-cell carcinomas. These may possibly represent secondary aberrations that influence the tumor differentiation pattern.

\section{Conclusions}

We have shown that aberrations such as gain of material from $8 \mathrm{q} 23$ and losses from 19q and 22q, previously described at high frequencies in bilateral and borderline ovarian carcinomas, are also present in tumors of more advanced stages, i.e., carcinomas; this highlights the possibility of an adenoma-carcinoma sequence in ovarian carcinogenesis. The low-grade carcinomas may represent a phenotypically more advanced stage of borderline tumors.

\section{Additional file}

Additional file 1: Table S1. Overview of the genomic aberrations found in carcinomas of the ovary, detected by karyotyping and HR-CGH.

\section{Competing interests}

The authors declare that they have no competing interests.

\section{Authors' contributions}

FM supervised all the experiments and interpreted the results. LH carried out the cytogenetic and molecular cytogenetic analysis. VMA and BD provided the samples and gave pathologic data. CGT provided clinical information. FM and $\mathrm{SH}$ wrote the paper. All authors read and approved the final manuscript.

\section{Acknowledgments}

This work was supported by grants from the Norwegian Cancer Society, the South-East Norway Regional Health Authority, and the Inger and John Fredriksen Foundation for Ovarian Cancer Research.

\section{Author details}

${ }^{1}$ Section for Cancer Cytogenetics, Institute for Cancer Genetics and Informatics, The Norwegian Radium Hospital, Oslo University Hospital, 0310 Oslo, Norway. ${ }^{2}$ Centre for Cancer Biomedicine, University of Oslo, Oslo, Norway. ${ }^{3}$ Department of Pathology, The Norwegian Radium Hospital, Oslo University Hospital, Oslo, Norway. ${ }^{4}$ Faculty of Medicine, University of Oslo, Oslo, Norway. ${ }^{5}$ Department of Gynecology, The Norwegian Radium Hospital Oslo University Hospital, Oslo, Norway.

Received: 7 January 2014 Accepted: 27 April 2014 Published: 5 May 2014

\section{References}

1. Tavassoli FA, Devilee P: World health organization classification of tumors: Pathology and genetics of tumors of the breast and female genital organs. Lyon: IARC press; 2003.

2. Blaustein A: Blaustein's Pathology of the Female Genital Tract. New York: Springer-Verlag; 2002.

3. Kurman RJ, Shih I: The origin and pathogenesis of epithelial ovarian cancer: a proposed unifying theory. Am J Surg Pathol 2010, 34:433-443.

4. Prat J: New insights into ovarian cancer pathology. Ann Oncol 2012, 23:111-117.
5. Mitelman F, Johansson B, Mertens F: Mitelman database of chromosome aberrations and gene fusions in cancer. 2014. http://cgap.nci.nih.gov/ Chromosomes/Mitelman

6. Micci F, Weimer J, Haugom L, Skotheim Rl, Grunewald R, Abeler VM, Silins I, Lothe RA, Trope CG, Arnold N, Heim S: Reverse painting of microdissected chromosome 19 markers in ovarian carcinoma identifies a complex rearrangement map. Gene Chromosome Canc 2009, 48:184-193.

7. Mandahl N: Methods in solid tumors. In Human Cytogenetics - a practical approach, vol II, Malignancy and acquired abnormalities. Edited by Rooney DE, Czepulkovski BH. Oxford: IRL Press; 1992:155-187.

8. Shaffer LG, Slovak ML, Campbell LJ: ISCN (2009): An International System for Human Cytogenetic Nomenclature. Basel: Karger S; 2009.

9. Brandal P, Bjerkehagen B, Heim S: Molecular cytogenetic characterization of tenosynovial giant cell tumors. Neoplasia 2004, 6:578-583.

10. Kallioniemi OP, Kallioniemi A, Piper J, Isola J, Waldman FM, Gray JW, Pinkel D: Optimizing comparative genomic hybridization for analysis of DNA sequence copy number changes in solid tumors. Gene Chromosome Canc 1994, 10:231-243.

11. Micci F, Teixeira MR, Haugom L, Kristensen G, Abeler VM, Heim S: Genomic aberrations in carcinomas of the uterine corpus. Gene Chromosome Canc 2004, 40:229-246.

12. Micci F, Haugom L, Ahlquist T, Andersen HK, Abeler VM, Davidson B, Trope CG, Lothe RA, Heim S: Genomic aberrations in borderline ovarian tumors. J Transl Med 2010, 8:21. doi:10.1186/1479-5876-8-21.

13. Onkes W, Fredrik R, Micci F, Schonbeck BJ, Martin-Subero JI, Ullmann R, Hilpert F, Brautigam K, Janssen O, Maass N, Siebert R, Heim S, Arnold N, Weimer J: Breakpoint characterization of the $\operatorname{der}(19) \mathrm{t}(11 ; 19)(q 13 ; p 13)$ in the ovarian cancer cell line SKOV-3. Gene Chromosome Canc 2013, 52:512-522.

14. Pejovic T, Heim S, Mandahl N, Baldetorp B, Elmfors B, Floderus UM, Furgyik S, Helm G, Himmelmann A, Willen H: Chromosome aberrations in 35 primary ovarian carcinomas. Gene Chromosome Canc 1992, 4:58-68.

15. Jenkins RB, Bartelt D Jr, Stalboerger P, Persons D, Dahl RJ, Podratz K, Keeney $G$, Hartmann L: Cytogenetic studies of epithelial ovarian carcinoma. Cancer Genet Cytogenet 1993, 71:76-86.

16. Thompson FH, Emerson J, Alberts D, Liu Y, Guan XY, Burgess A, Fox S, Taetle $\mathrm{R}$, Weinstein R, Makar R: Clonal chromosome abnormalities in 54 cases of ovarian carcinoma. Cancer Genet Cytogenet 1994, 73:33-45.

17. Micci F, Skotheim RI, Haugom L, Weimer J, Eibak AM, Abeler VM, Trope CG, Arnold N, Lothe RA, Heim S: Array-CGH analysis of microdissected chromosome 19 markers in ovarian carcinoma identifies candidate target genes. Gene Chromosome Canc 2010, 49:1046-1053.

18. Micci F, Haugom L, Ahlquist T, Abeler VM, Trope CG, Lothe RA, Heim S: Tumor spreading to the contralateral ovary in bilateral ovarian carcinoma is a late event in clonal evolution. J Oncol 2010, 2010:646340. doi:10.1155.

19. Iwabuchi H, Sakamoto M, Sakunaga H, Ma YY, Carcangiu ML, Pinkel D, YangFeng TL, Gray JW: Genetic analysis of benign, low-grade, and high-grade ovarian tumors. Cancer Res 1995, 55:6172-6180.

20. Arnold N, Hagele L, Walz L, Schempp W, Pfisterer J, Bauknecht T, Kiechle M: Overrepresentation of $3 q$ and $8 q$ material and loss of $18 q$ material are recurrent findings in advanced human ovarian cancer. Gene Chromosome Canc 1996, 16:46-54.

21. Sonoda G, Palazzo J, Du MS, Godwin AK, Feder M, Yakushiji M, Testa JR: Comparative genomic hybridization detects frequent overrepresentation of chromosomal material from $3 q 26,8 q 24$, and $20 q 13$ in human ovarian carcinomas. Gene Chromosome Canc 1997, 20:320-328.

22. Tapper J, Sarantaus L, Vahteristo P, Nevanlinna H, Hemmer S, Seppala M, Knuutila S, Butzow R: Genetic changes in inherited and sporadic ovarian carcinomas by comparative genomic hybridization: extensive similarity except for a difference at chromosome 2q24-q32. Cancer Res 1998, 58:2715-2719.

23. Suzuki S, Moore DH, Ginzinger DG, Godfrey TE, Barclay J, Powell B, Pinkel D, Zaloudek C, Lu K, Mills G, Berchuck A, Gray JW: An approach to analysis of large-scale correlations between genome changes and clinical endpoints in ovarian cancer. Cancer Res 2000, 60:5382-5385.

24. Kiechle M, Jacobsen A, Schwarz-Boeger U, Hedderich J, Pfisterer J, Arnold N: Comparative genomic hybridization detects genetic imbalances in primary ovarian carcinomas as correlated with grade of differentiation. Cancer 2001, 91:534-540.

25. Cancer Genome Atlas Research Network: Integrated genomic analyses of ovarian carcinoma. Nature 2011, 474:609-615. 
26. Mayr D, Hirschmann A, Lohrs U, Diebold J: KRAS and BRAF mutations in ovarian tumors: a comprehensive study of invasive carcinomas, borderline tumors and extraovarian implants. Gynecol Oncol 2006, 103:883-887.

27. Ardighieri L, Zeppernick F, Hannibal CG, Vang R, Cope L, Junge J, Kjaer SK, Kurman RJ, Shih IM: Mutational analysis of BRAF and KRAS in ovarian atypical proliferative serous (Borderline) tumors and associated peritoneal implants. J Pathol 2013, 232:16-22.

28. Tsang YT, Deavers MT, Sun CC, Kwan SY, Kuo E, Malpica A, Mok SS Gershenson DM, Wong KK: KRAS (but not BRAF) mutations in ovarian serous borderline tumor are associated with recurrent low-grade serous carcinoma. J Pathol 2013, 231:449-456.

doi:10.1186/1471-2407-14-315

Cite this article as: Micci et al:: Genomic profile of ovarian carcinomas. BMC Cancer 2014 14:315.

\section{Submit your next manuscript to BioMed Central and take full advantage of:}

- Convenient online submission

- Thorough peer review

- No space constraints or color figure charges

- Immediate publication on acceptance

- Inclusion in PubMed, CAS, Scopus and Google Scholar

- Research which is freely available for redistribution 